\title{
USO DE HABITATS NATURAIS E ANTRÓPICOS PELOS ANUROS EM UMA LOCALIDADE NO ESTADO DE RONDÔNIA, BRASIL (AMPHIBIA: ANURA)
}

\author{
Paulo Sérgio BERNARDE', Marcelo Nogueira de Carvalho KOKUBUM², \\ Reginaldo Assêncio MACHADO' ${ }^{1}$, Luiz dos ANJOS ${ }^{3}$
}

RESUMO - Neste trabalho é apresentado a lista de espécies de anfibios anuros da Fazenda Jaburi, Municipio de Espigão D’Oeste, Estado de Rondônia, enfocando os ambientes utilizados durante a atividade reprodutiva em áreas de floresta primária e campos de pastagens. Foram registradas 31 espécies para esta localidade pertencentes as seguintes familias: Bufonidae (3), Dendrobatidae (2), Hylidae (17), Leptodactylidae (7) e Microhylidae (2). A maioria das espécies (24) ocorreram no interior e na borda de floresta primária. Quatorze espécies foram observadas em atividade reprodutiva nos campos de pastagens, enquanto nove espécies foram encontradas apenas dentro de floresta primária.

Palavras-chave: anfibios anuros, habitats, Amazônia

Utilization of Natural and Antropogenic Habitats by Anurans at One Locality in Rondônia State, Brazil (Amphibia: Anura)

ABSTRACT - This paper presents a list of anurans species from Jaburi farm, Espigão D'Oeste Municipality, with special focus on the habitats used during the reproduction period in primary forest and pasture areas. Thirty one species were recorded for this locality from the following families: Bufonidae (3), Dendrobatidae (2), Hylidae (17), Leptodactylidae (7) and Microhylidae (2). The majority of species (24) occurred into and around the primary forest. Fourteen species were observed in breeding activity in the pasture areas, while 9 species were finded only inside primary forest.

Key-words: anuran amphibians, habitats, Amazon

\section{INTRODUÇÃO}

A Amazônia representa uma das últimas áreas florestais pouco modificadas pela ação humana na Terra, sendo de extrema importância o estudo de sua biodiversidade (Overal \& Mascarenhas, 1993; Gascon \& Moutinho, 1998). Em relação à anurofauna, são poucos os trabalhos sobre comunidades neste bioma (Duellman, 1990). O mesmo panorama de escassez de informações também ocorre na Amazônia brasileira, onde poucas localidades foram amostradas (e. g., Crump, 1971; Hodl, 1977; Zimmerman \& Bierregaard, 1986; Zimmerman \& Rodrigues, 1990; Gascon \& Pereira, 1993; Tocher, 1998). Informações sobre história natural e ecologia reprodutiva da maioria das espécies são ainda mais raras (Gascon, 1995).

No Estado de Rondônia, destacase o estudo de Heyer (1977) em algumas localidades ao longo do rio

1. Universidade Federal do Paraná, Departamento de Zoologia, Curso de Pós-Graduação em Zoologia. Caixa Postal 19020, 81531-990, Curitiba, Paraná.

2. Univ. Fed. Uberlândia, Dept. de Biociências. Curso de Pós-Graduação em Ecologia e Conservação de Recursos Naturais. Caixa Postal 593. 38400-902 Uberlândia, MG

3. Universidade Estadual de Londrina, Departamento de Biologia Animal e Vegetal. Caixa Postal 6001. 86051-970, Londrina, Paraná. 
Madeira, e o de Vanzolini (1986), que apresenta uma lista de espécies coletadas ao longo da $\mathrm{Br}-364$ durante o Projeto Polonoroeste, região esta que está sofrendo transformações com a colonização.

No presente trabalho é apresentado uma lista de espécies de anfibios anuros em uma localidade no Estado de Rondônia, fornecendo os ambientes utilizados durante a atividade reprodutiva.

\section{MATERIAL E MÉTODOS}

Os trabalhos de campo foram realizados na Fazenda Jaburi (11\%3511 $\left.938 \mathrm{~S} ; 60^{\circ} 41-60^{\circ} 45 \mathrm{~W}\right)$, localizada no municipio de Espigão D'Oeste, Estado de Rondônia. A Fazenda, assim como outras áreas, vem sendo colonizada recententemente, região esta que era originalmente caracterizada por florestas e ausẻncia de áreas abertas naturais. Nesta localidade o desmatamento teve inicio a cerca de 15 anos atrás, apresentando atualmente 2500 hectares de áreas de florestas primárias (Floresta Ombrófila Aberta) e 1500 hectares de áreas abertas (campos de pastagens).

A altitude da região varia em torno de 540 metros. Apresenta uma média pluviométrica de $2100 \mathrm{~mm}$ anuais, compreendendo o periodo de seca entre abril a setembro e a temperatura média de $30^{\circ} \mathrm{C}$, sendo a minima de $20^{\circ} \mathrm{C}$ e a máxima de $38^{\circ} \mathrm{C}$ (dados fornecidos pela Prefeitura Municipal de Espigão D'Oeste).

Cinco viagens a esta localidade foram realizadas, cada uma com duração de cerca de 10 dias, nos seguintes meses: julho de 1995 , janeiro de 1996, janeiro de 1997, julho de 1997 e janeiro de 1998 . Os habitats foram classificados segundo McDiarmid (1994): terrestres, em relação a cobertura vegetacional (floresta, borda de floresta e área de pastagens); aquáticos, em relação a velocidade da água (lênticos e lóticos) e durabilidade da poça (permanentes e temporários). Os habitats percorridos foram: no interior de floresta, poças permanentes e temporárias (na época das chuvas), riachos (menos de $2 \mathrm{~m}$ de largura e menos de $0,50 \mathrm{~m}$ de profundidade) e ribeirão (mais de $4 \mathrm{~m}$ de largura e mais de $0,50 \mathrm{~m}$ de profundidade); nas áreas abertas e de borda de floresta, além desses corpos d'água, foram percorridos tambèm açudes construidos como bebedouros para o gado.

Para o levantamento das espécies e obtenção dos dados sobre a distribuição das mesmas nos habitats, foi seguido a técnica de Scott Jr. e Woodward (1994), onde foram percorridos todos os ambientes (durante o dia e principalmente a noite) acima citados e anotadas todas as espécies em atividade de vocalização. Durante cada fase, os esforços de amostragem foram similares para todos os habitats.

Para a identificação das espécies foram utilizadas as chaves e descrições de Bokermann (1963), Lutz (1973), Duellman (1978), Hero (1990) e por comparação com espécimes depositados na coleção do Museu de 
História Natural da Universidade Estadual de Campinas (ZUEC), São Paulo. Espécimes testemunhos estão depositados nas seguintes coleçôes científicas: Coleção Herpetológica da Universidade Estadual de Londrina (MZUEL) (Londrina, Paraná), Museu de História Natural Capão da Imbuia (MHNCI) (Curitiba, Paraná) e ZUEC (ver relação em Apêndice).

\section{RESULTADOS E DISCUSSÃO}

Um total de 31 espécies de anfibios anuros foram registradas na Fazenda Jaburi, das quais 29 foram observadas em atividade de vocalização (Tab, 1). Bufo marinus (Linnaeus, 1758) (Bufonidae) e Osteocephalus sp. (Hylidae) não foram encontradas reproduzindo-se. Espera-se um número maior de espécies na localidade devido à riqueza da anurofauna amazónica (ver Duellman, 1990). Na região de Manaus, Amazonas, onde a anurofauna foi bem estudada nestes últimos anos (Zimmerman \& Bierregaard, 1986; Zimmerman \& Rodrigues, 1990; Gascon, 1991), são conhecidas 62 espécies de anuros (Tocher, 1998), O baixo número de espécies encontrado neste estudo provavelmente se deva ao fato desta localidade só ter sido amostrada durante os meses de janeiro e julho e não sazonalmente, Vanzolini (1986) havia registrado 70 espécies de anuros para o Estado de Rondônia. Seis espécies (Colostethus brunneus, Hyla microcephala, Osteocephalus taurinus, Phyllomedusa tarsius, Proceratophrys sp.n. e Elachistocleis sp.), não foram citadas em Vanzolini (1986)

$\mathrm{O}$ interior de floresta primária e sua borda foram os ambientes mais ricos em numero de espécies (ambos com 17 cada), enquanto na área aberta foram encontradas 14 espécies. O maior número de espécies encontrado na área de floresta deve-se provavelmente à maior heterogeneidade ambiental, conforme mencionado por Pianka (1967). Portanto, dentro de floresta primária, onde existe maior disponibilidade de ambientes para reprodução (sítios de vocalização e oviposição), pode ser encontrado um maior: número de espécies (Cardoso et al., 1989).

Quatorze espécies foram encontradas em atividade reprodutiva (vocalizando; presença de girinos, imagos e casais em amplexo) nos campos de pastagens, podendo ser consideradas como residentes (ver Tocher, 1998). Estas espécies reproduziram-se principalmente nos açudes permanentes e poças temporárias (Tab.1). As duas espécies do gênero Adenomera e Eleutherodactylus fenestratus apresentam reproduçâo terrestre (Duellman, 1978) e foram encontrados vocalizando nas bases das touceiras de capins, as quais aparentemente são capazes de reter umidade.

As sete espécies que foram encontradas apenas dentro de floresta (Tab. 1), parecem ser mais sensiveis ao desmatamento. As duas espécies de dendrobatideos são diurnas e vivem na serapilheira de florestas (Duellman, 1978). Osteocephalus taurinus e Phyllomedusa tomopterna utilizam o estrato arbóreo durante a vocalização (Duellman, 1978; 1990). Hamptophryne boliviana e Leptodactylus pentadactylus são 
Tabela 1. Ocorrência das espécies de anuros nos ambientes durante a reprodução na Fazenda Jaburi, Espigão D'Oeste - RO, FLOR = interior de floresta, BORDA = borda de floresta, PASTO $=$ campo de pastagem, $\mathrm{pp}=$ poças permanentes, $\mathrm{pt}=$ poças temporárias, $\mathrm{ri}=$ riachos, $\mathrm{ld}=$ locais distantes de corpos d'água, $a c ̧=$ açudes, $b r=$ brejo.

\begin{tabular}{|c|c|c|c|}
\hline ESPECIE & FLOA & BORDA & PASTO \\
\hline \multicolumn{4}{|l|}{ Bufonidae } \\
\hline Buto guttatus Schneider, 1799 & ri & ri & \\
\hline Bufo gr. typhonius & ri & ri & \\
\hline \multicolumn{4}{|l|}{ Dendrobatidae } \\
\hline Colostethus brunneus (Cope, 1887) & pt, ri, ld & & \\
\hline Colostethus gr. marchesianus & $\rho t$, ri, ld & & \\
\hline \multicolumn{4}{|l|}{ Hylid ae } \\
\hline Hyla bokerm anni Goin, 1960 & & pt & \\
\hline Hyla fasciata Gunther, 1859 & & pt & \\
\hline Hyla geographica Spix, 1824 & & $p p, r i$ & $p p$ \\
\hline Hyla lanciform is (Cope, 1870) & $p p, r i$ & $p p, r i$ & \\
\hline Hyla leucophyllata (Beireis, 1783) & & $p p, p t$ & $p p, p t$ \\
\hline Hyla microcephala Cope, 1886 & & pt & pt \\
\hline Hyla m inuta Peters, 1872 & & & pt \\
\hline Hyla multifasciata Gunther, 1859 & & & br \\
\hline Hyla punctata (Schneider, 1799) & $p t$ & $\mathrm{pt}$ & \\
\hline Hyla schubartii Bokermann, 1963 & $p p$ & $\mathrm{pt}$ & \\
\hline Osteocephalus taurinus Steindachner, 1862 & pt & & \\
\hline Phyllom edusa tarsius (Cope, 1868) & $p p, p t$ & $p p, p t$ & pt \\
\hline Phyllomedusa tomopterrna (Cope, 1868) & $p p$ & & \\
\hline Phyllom edusa vaillanti Boulenger, 1882 & $p p$ & $\mathrm{pp}$ & \\
\hline Scinax rubrus (Laurenti, 1768) & & & $p p, p t$ \\
\hline Scinax nebulosus (Spix, 1824) & & $p p$ & $\rho \rho$ \\
\hline \multicolumn{4}{|l|}{ Lep to d actylid ae } \\
\hline Adenomera andreae (Müller, 1923) & Id & Id & Id \\
\hline Adenomerasp. & Id & Id & Id \\
\hline Eleutherodactylus fenestratus (Steindachner, 1864) & Id & Id & Id \\
\hline Leptodactylus fuscus (Schneider, 1799) & & & pt \\
\hline Leptodactylus pentadactylus (Laurenti, 1768) & pt & & \\
\hline Physalaemus petersi (Espada, 1872) & & $p p, p t$ & $p p$ \\
\hline Proceratophrys sp. & $p p$ & & \\
\hline \multicolumn{4}{|l|}{ Microhylidae } \\
\hline Elachistocleis sp. & & & pt \\
\hline Hamptophryne boliviana (Parker, 1927) & $\mathrm{pt}$ & & \\
\hline Total de Espécies & 17 & 17 & 14 \\
\hline
\end{tabular}


espécies associadas a florestas (Zimmerman \& Rodrigues, 1990; Rodriguez \& Cadle, 1990). A nova espécie de Proceratophrys que está em fase de descrição (Giaretta et al., no prelo), foi encontrada apenas dentro de floresta, o que é usual para as espécies do gênero (Heyer et al., 1990). Provavelmente as exigências dessas espécies em relação aos microambientes para a reprodução, as tornam inaptas à colonização das áreas alteradas (ver Tocher, 1998).

Zimmerman \& Bierregaard (1986) mostraram a importância do tamanho de áreas de florestas primárias na manutenção da anurofauna na região Central da Amazổnia, analisando a composição de espécies em fragmentos florestais de diversos tamanhos. Tocher (1998) estudando áreas com diferentes graus de perturbação (floresta primária, florestas secundárias e campos de pastagens), encontrou um nítido decréscimo no número de espécies entre a floresta primária e os campos de pastagens. Uma das causas do menor número de espécies de anuros em campos de pastagens em relação a floresta primária está relacionada com a perda dos ambientes utilizados para a reprodução (Tocher, 1998). Além disso, algumas espécies de anuros que ainda se reproduzem em áreas abertas, podem depender da floresta para sobreviverem durante a estação não reprodutiva. Como é o caso de Hyla faber na região sudeste do Brasil (Márcio Martins, com. pess.).

Grande parte do Estado de
Rondônia sofreu e vem sofrendo impacto devido à recente colonização (Vanzolini, 1986). Na região, está ocorrendo intenso desmatamento para a retirada de madeira e formação de pastagens, o que alėm da destruição da floresta (Gascon \& Moutinho, 1998), também pode causar mudanças climáticas (Myers, 1997). Sabe-se que vários tipos de deteriorações ambientais estão causando extinções em anuros (Heyer et al,, 1988; Weygoldt, 1989), no presente trabalho foi apontado algumas espécies que não encontram condições adaptativas às areas de campos de pastagens. Por isso è salientado a relevância da legislação brasileira em proibir o desmatamento de $50 \%$ da área das propriedades no estado e em torno dos rios, sendo crucial para a conservação da anurofauna desta região. Outra medida de conservação, seria a escolha da localização da reserva legal dentro da propriedade, que poderia ser contígua com a da propriedade vizinha, constituindo uma área florestal de maior tamanho. A importância destas medidas em relação a anurofauna é o fato de que na Amazônia, quanto maior o tamanho do fragmento florestal, maior é o número de espécies que neles podem ser encontrados (Zimmerman \& Bierregaard, 1986; Tocher, 1998).

\section{AGRADECIMENTOS}

Somos gratos a Ariovaldo A. Giaretta pelo auxílio na identificação de algumas espécies, pela leitura do manuscrito e acesso a ZUEC. A Sérgio 
Augusto A. Morato pela leitura do manuscrito. A Júlio C. de Moura Leite, Márcia F. Renner e Ulisses Albino no auxílio em algumas coletas. A Eduardo Garcia e familia pelo auxílio prestado e por permitir a pesquisa em sua propriedade. AArlindo Bernarde Liberal pelo auxilio prestado. A Capes e ao $\mathrm{CNPq}$ pelas bolsas concedidas à $\mathrm{PSB}$ e RAM, respectivamente. LdA dispõe de bolsa produtividade em pesquisa do $\mathrm{CNPq}$.

\section{Bibliografia citada}

Bokermann, W,C,A. 1963. Duas novas espécies de "Hyla"de Rondônia. Brasil (Amphibia, Salientia). Rev, Bras. Biol. 23: $247-250$.

Cardoso, A.J.; Andrade, G.V.; Haddad, C.F.B. 1989. Distribuição espacial em comunidades de anfibios (Anura) no sudeste do Brasil. Rev: Bras. Biol., 49: 241-249.

Crump, M.L. 1971. Quantitative analysis of the ecological distribution of a tropica! herpetofauna. Occas. Pap, Mus. Nat. Hist. Univ: Kansas, 3: 1-62.

Duellman, W.E. 1978. The biology of na equatorial herpetofauna in Amazonian Equador. Univ. Kansas Misc. Publ., 65: $1-352$.

Duellman, W.E. 1990. Herpetofaunas in Neotropical Rainforests: comparative composition, history, and resource use, In: Gentry, A.H. (Ed), Four Neotropical Rainforests, New Haven, Connecticut, Yale University Press, p. 455-505.

Gascon, C. 1991. Population and community level analyses of species occurrences of central Amazonian rainforest tadpoles. Ecology, 72: 1731-1746.

Gascon, C. 1995. Natural history notes on frogs from Manaus, Amazonas. Revta Bras. Zool., 12: 9-12.
Gascon, C.; Moutinho, P. 1998. Floresra Amazônica: Dinâmica, Regeneração e Manejo. Manaus, Amazonas, Ministério da Ciência e Tecnologia, Instituto de Pesquisa da Amazonnia, 373p.

Gascon, C.; Pereira, O.S.P. 1993. Preliminary checklist of the herpetofauna of the upper Rio Urucu, Amazonas, Brazil. Revia Bras. Zool., 10: 179-183.

Giaretta, A. A.; Bernarde, P. S.; Kokubum, M. C. N. (no prelo). A new species of Proceratophrys (Anura: Leptodactylidae) from the Amazon Rain Forest. $J$. Herpetol.

Hero, J.M. 1990. Na illustrated key to tadpoles occurring in the Central Amazonian rainforest, Manaus, Amazonas, Brasil. Amazoniana, 11: 201-262.

Heyer, W.R, 1977. Taxonomic notes on frogs from the Madeira and Purus rivers, Brasil. Papéis Avulsos Zool., 31: 141-162

Heyer, W.R.; Rand, A.S.; Cruz, C.A.G.. Peixoto, O.L. 1988. Decimations, extincions, and colonizations of frog populations in Southeast Brazil and their evolutionary implications. Biotropica. 20: 230-235.

Heyer, W.R.; Rand, A.S.; Cruz, C,A.G.; Peixoto, O.L.; Nelson, C.E. 1990. Frogs of Boracéia. Arq. Zool., 31:231-410.

Hodl, W. R. 1977. Call differences and calling site segregation in anuran species from Central Amazonian floating meadows. Decologia, 28: 351-363.

Lutz, B. 1973. Brazilian species of Hyla. University of Texas Press: Austin and London, $265 p$.

McDiarmid, R.W. 1994. Keys to a successful project: associated data and planning: Data Standards. In: Heyer, W.R.; Donnelly, M.A.; Mcdiarmid, R.W.; Hayek, L.C.; Foster, M.S. (Eds). Measuring and Monotoring Biological Diversity. Standard Methods for Amphibians. Washington, Smithsonian Institution Press, p.57-60.

Myers, N. 1997. Florestas tropicais e suas 
espécies sumindo, sumindo...? In: Wilson, E.O. (Org). Biodiversidade, Editora Nova Fronteira, Rio de Janeiro-RJ, p. 36-45.

Overal, W.L.; Mascarenhas, B.M. 1993. Recomendaçôes para 0 inventảio faunistico da Amazônia. Bol. Mus. Par. Emílio Goeldi Sèr: Zool., 9: 329-339.

Pianka, E.R. 1967. On lizard species diversity: North American flatand deserts. Ecology, 48: 333-351.

Rodriguez, L.B.; Cadle, J.E. 1990. A preliminary overview of the herpetofauna of Cosha Cashu, Manu National Park, Peru. In: Gentry, A. H. (Ed). Four Neotropical Rainforests. New Haven, Connecticut, Yale University Press, p. 410-425.

Seott Jr., N.J.; Woodward, B.D. 1994. Standard techniques for inventory and monitoring: Surveys at Breeding Sites. In: Heyer, W.R.; Donnelly, M.A.; Mcdiarmid, R.W.; Hayek, L.C.; Foster, M.S. (Eds). Measuring and Monotoring Biological Diversity. Standard Methods for Amphibians. Washington. Smithsonian Institution Press, p. 118-125.

Tocher, M. 1998. Diferenças na composição de espécies de sapos entre três tipos de floresta e campo de pastagem na Amazônia central. In: Gascon, C.; Moutinho, P. (Eds). Floresta Amazônica: Dinâmica, Regeneração e Manejo. Amazonas, Manaus, Ministério da Teconologia e Ciência, Instituto de Pesquisas da Amazônia, p. 219-232.

Vanzolini, P.E. 1986. Levantamento herpetológico da área do Estado de Rondônia sob influència da rodovia $B R$ 364. Relatório de Pesquísa $\mathrm{n}^{2} 1$. Brasilia, CNPq, Assessoria Editorial, 50p.
Weygoldt, P. 1989. Changes in the composition of mountain stream frog commnuties in the Atlantic mountains of Brazil: Frogs as indicators of environmental deteriorations? Studies on Neotropical Fauna and Environment, 243: 249-255.

Zimmerman, B.L.; Bierregaard, R.O. 1986. Releyance of the equilibrium theory of island biogeography and species-area relations to conservation with a case from Amazonia. J. Biogeogr, 13: 133143.

Zimmerman, B.L.; Rodrigues, M.T. 1990. Frogs, snakes, and lizards of the INPAWWF reserves near Manaus, Brazil $l n$; Gentry, A.H. (Ed). Four Neotropical Rainforests. New Haven, Connecticut. Yale University Press, p. 426-454. 


\section{APÊNDICE}

Espécimes testemunhos colecionados: Bufo guttatus (MZUEL 463, 1028, 1029, 1030, 1031, 1032, 1033); Bufo marinus (MZUEL 338, 339); Bufo gr. typhonius (MZUEL 333, 1034); Colostethus brunneus (MZUEL 443, 963, 964 , 965, 974, 975, 976, 977, 978, 986, 987, 988, 989, 990, 991, 992, 1113, 1114, 1115; MHNCI); Colostethus gr. marchesianus (MZUEL 332, 444, 445, 446, 962); Hyla bokermanni (MZUEL 973); Hyla fasciata (MZUEL 899, 900, 901; MHNCI 3331, 3335); Hyla geographica (MZUEL 457, 458, 459, 464, 468, 902 , 903, 904; MHNCI 3005, 3330); Hyla lanciformis (MZUEL 908); Hyla leucophyllata (MZUEL 456, 895, 898, 957, 958); Hyla microcephala (MZUEL $890,891,892,893,894,896,897,959,960,971,972$ ); Hyla minuta (MZUEL 966, 967, 968, 980; MHNCI 3592); Hyla multifasciata (MZUEL 469, 905, 906, 907); Hyla punctata (MHNCI 3590); Hyla schubarti (MZUEL 981, 982; MHNCI 3321, 3327); Osteocephalus taurinus (MZUEL 351; MHNCI 3320, 3585); Osteocephalus sp. (MZUEL 447); Phyllomedusa tarsius (MZUEL 925, 926, 927; MHNCI 3322, 3323, 3324, 3325, 3326); Phyllomedusa tomopterna (MHNCI 3587); Phyllomedusa vaillanti (MZUEL 360, 361, 455; MHNCI 3588); Scinax rubra (MZUEL 336, 340, 341, 343, 345, 346, 347, 348, 349, $350,352,353,354,355,356,358,359,482$; MHNCI 3007, 3008, 3009, 3334); Scinax nebulosus (MZUEL 451, 453, 454, 480, 481, 912, 913, 914, 915, 916, 917, 918, 919, 920, 921, 922, 923, 924; MHNCI 3010; ZUEC 11376, 11377, 11378, 11379, 11380); Adenomera andreae (MZUEL 472, 473); Adenomera sp. (MZUEL 961, 1036, 1037); Eleutherodactylus fenestratus (MZUEL 334, 335, $442,448,449,450,470,471,479,485,937,938,939,940,941,942,943,944$, $945,946,947,948,949,950,951,952,953,970,983,985,1116,1120,1121$, 1122, 1123, 1124; MHNCI 3333, 3586); Leptodactylus fuscus (MZUEL 928, 929, 930, 931); Leptodactylus pentadactylus (MZUEL 932, 933, 934, 935, 954); Physalaemus petersi (MZUEL 955, 956, 969); Proceratophrys sp. (MZUEL 936; ZUEC 11387, 11388, 11389, 11390); Elachistocleis sp. (MZUEL 641; MHNCI 3328, 3332, 3336; ZUEC 11381, 11382, 11383, 11384, 11385, 11386); Hamptophryne boliviana (MHNCI 3329, 3589). 